\title{
PHYSICAL POSSIBILITY AND DETERMINATE NUMBER THEORY
}

\author{
SHARON BERRY
}

\begin{abstract}
It's currently fashionable to take Putnamian modeltheoretic worries seriously for mathematics, but not for discussions of ordinary physical objects and the sciences. However, I will argue that (under certain mild assumptions) merely securing determinate reference to physical possibility suffices to rule out the kind of nonstandard interpretations of our number talk Putnam invokes. So, anyone who accepts determinate reference to physical possibility should not reject determinate reference to the natural numbers on Putnamian model-theoretic grounds 1
\end{abstract}

\section{INTRODUCTION}

In [17] and [16] Putnam uses the existence of unintended models for our best scientific and mathematical theories to raise a challenge for our ability refer to physical objects or a single intended natural number structure. It's currently fashionable to take such model-theoretic doubts about reference seriously in the mathematical case but assume that analogous worries about the reference for physical vocabulary can somehow be answered 2 ,

\footnotetext{
${ }^{2}$ For example, this position is discussed in $3,10,14$. I find this combination of attitudes is even more often held implicitly and acknowledged under questioning than explicitly defended in print. Philosophers are often willing to advance Putnamian model-theoretic arguments as reasons to doubt our ability to successfully refer to intended mathematical structures, while feeling free to ignore them as far as talk of physical objects and the necessity relevant to physical laws is concerned.
} 
In this paper, I will attack this combination of views. I will argue that if we grant the realist determinate reference to certain physical notions (including a notion of physical possibility ${ }^{3}$ ) and a certain physical assumption holds, they can block Putnam's model theoretic argument that we can't determinately refer to the standard model of the natural numbers. So, philosophers who believe our physical vocabulary and notion of physical possibility successfully refer should reject Putnam's argument that we can't uniquely refer to the natural numbers 4

In section 2 I will set up the model-theoretic challenge to mathematical realism I want to consider. In section 3 I'll review some limitations and problems for previous work on (approximately) this topic by Hartry Field and Vann McGee. In section 4 I will spell out my main argument and finally in section 5 I will discuss some helpful variants on this argument.

\footnotetext{
${ }^{3}$ My argument would not work without this assumption of definite reference to physical possibility, for the reasons Putnam points out on pages 469-470 of [17]. He notes that merely citing our acceptance of certain counterfactual sentences don't suffice to block putnaminan unintended interpretation as follows, "Suppose we 'first orderize' counterfactual talk, say, by including events in the ontology of our theory and introducing a predicate ('subjunctively necessitates') for the counter-factual connection between unactualized event types at a given place-time. Then our argument shows that a model exists which fits all the facts that will actually be registered or observed and fits our theoretical constraints, and this model induces an interpretation of the counterfactual idiom (a 'similarity metric on possible worlds', in David Lewis' theory) which renders true just the counterfactuals that are true according to some completion of our theory."

${ }^{4}$ Note that I'm not arguing that the combination of, so to speak, physical determinacy and number-theoretic indeterminacy is itself incoherent. I only claim to show that if we assume determinate reference for certain pieces of physical vocabulary then one specific (famous) argument that we can't secure determinate reference to the natural number structure is blocked.
} 


\section{Putnam's Model-Theoretic Challenge(s)}

\subsection{A Challenge for Physical and Mathematical Realists. Let's}

begin by considering Putnam's model-theoretic challenge, as it applies to the natural numbers.

From a naive realist point of view, it appears that we can refer to the natural numbers (at least up to isomorphism). A natural place to look for an explanation of our ability to refer to the natural numbers is our beliefs about them. Thus, one might hope to appeal to our acceptance of various statements of first-order number theory (plus our grasp of various first-order logical connectives) to explain how our number talk secures definite reference.

However, by the Lowenhiem-Skolem theorem, any first-order theory which has an infinite model (as our first-order number theory must), has models of different sizes. Thus, our mere beliefs about number theory and grasp of first-order logical vocabulary cannot pin down reference to the natural numbers. For example, the standard first-order Peano Axioms of arithmetic (PA) plausibly articulate part of our concept of numbers. But these axioms can also be satisfied by non-standard models.

In light of the existence of such non-standard models, one can ask (as Putnam does) the following question. Do we really have a definite concept of the structure of the natural numbers, which is not satisfied by the non-standard models? What can such a concept consist of? What is it about us which (perhaps together with other kinds of facts about the world) lets our words like "number" and "plus" take on 
meanings which rule out non-standard models? For reasons I won't discuss here, Putnam takes our ability to give standard meanings to first-order logical vocabulary for granted in this challenge. I will follow him in doing so.

Accordingly, we can dramatize Putnam's challenge as follows. Imagine an all-knowing perverse interpreter, who is dedicated to interpreting our talk about the natural numbers in some unintended fashion while preserving the meaning of our first-order logical vocabulary. And suppose (as Putnam does in formulating his challenge) that this perverse interpreter is free to use all of ordinary mathematics in their effort to construct non-standard models for our talk of the natural numbers to refer to.

Can we cite plausible constraints that our perverse interpreter must honor which prevent her from giving an unintended interpretation of our word 'number'? Note that, by the Löwenheim-Skolem theorem mentioned above, merely requiring she interpret first-order logical vocabulary normally and make some set of first order axioms about the natural numbers come out true (e.g., axioms extending PA or embedding the numbers in a larger structure) couldn't provide such a constraint.

If we can give no satisfying answer to Putnam's challenge then, perhaps, we must give up the realist intuition that we can refer to the standard model of the natural numbers. For example, we might allow that our conception of the structure of the natural numbers is vague and allows for a range of acceptable precisifications (corresponding to 
different non-isomorphic structures satisfying at least the Peano Axioms), analogous to the range of different acceptable personifications of 'bald' and 'heap ${ }^{\sqrt{5}}$.

Failure to answer Putnam's challenge also raises a problem for the common presumption that all statements of arithmetic (even ones we can't decide) have definite truth-values. For one thing, the most common way of accounting for such definite truth-values is through definite reference to the natural numbers. But, more directly, combining Gödel's Completeness and Incompleteness theorems [8 tells us that any computably axiomatizable theory extending basic arithmetic (a subsystem of Peano Arithmetic) — such as our first-order mathematical beliefs — will have models which disagree on the truth of some number-theoretic claims. So, if we concede to the Putnamian skeptic that every model of our first-order mathematical beliefs is an equally acceptable precisification of our number concept, then we are forced to also conclude that the truth-value of some number-theoretic claim is indeterminate.

Of course, if one took our ability to employ full second-order quantification (as the realist understands it) for granted, one could use our acceptance of second-order Peano Arithmetic (a version of Peano Arithmetic which replaces the first-order induction axiom schema with the second-order induction axiom below) to explain what is wrong with non-standard models of the natural numbers.

Induction Axiom: $(\forall X)[(X(0) \wedge(\forall n)(X(n) \rightarrow X(S(n)))) \rightarrow(\forall m) X(m)]$

${ }^{5}$ C.f. 7 . 
For the non-standard models of PA guaranteed by Löwenheim-Skolem and completeness arguments (discussed above) don't satisfy this induction axiom, if we take second-order quantification to range over all possible subsets. However, our ability to pin down standard meanings for second-order quantifiers is (prima facie) mysterious in just the same ways as our ability to latch on to the standard model of the natural numbers. So, this fact is not enough to banish Putnamian skeptical worries on its own.

Putnam supplements this mathematics-specific worry with a more general model-theoretic challenge to realist approaches to truth and reference. Very crudely, the worry goes like this. From a naive realist point of view, it seems that we can talk about physical objects and grasp scientific concepts in a way that makes it possible for an ideal scientific theory ${ }^{6}$ to be wrong. However, any consistent first-order theory can be interpreted as speaking truly about (some of) the sets. So why doesn't our ideal scientific theory count as speaking truly of this model? Why isn't it more charitable to interpret us as speaking truly of some of the sets rather than falsely of electrons and rabbits (and the rest of the apparent subject matter of the theory)?

As noted above, many philosophers are inclined to take Putnam's model-theoretic worries about determinate reference in mathematics seriously, while presuming that corresponding worries about reference to physical objects and notions can somehow be answered. Perhaps this difference in attitudes can be motivated by the abstractness of

\footnotetext{
${ }^{6}$ By 'an ideal theory', I mean a theory that would be accepted 'in the ideal limit' of scientific investigation.
} 
mathematical objects. One can invoke causal contact with the physical objects we seemingly refer to (cats and rabbits and electrons and so forth) to help explain definite reference to physical objects. But, since mathematical objects are generally taken to be causally inert, we cannot do the same when answering Putnam's challenge regarding reference to mathematical objects.

I will criticize this combination of views in what follows. I'll argue that (given certain mild physical assumptions) if we have definite reference to certain physical notions (including physical possibility), Putnam's skeptical argument about the natural numbers is blocked.

2.2. Clarifying the Challenges. But first let me make a quick remark about how I am understanding the Putnamian challenges introduced above, and what it would take to answer them.

Like most philosophically interesting skeptical arguments, the Putnamian challenge I'm concerned with in this paper doesn't just highlight the possibility of doubting some realist doctrine. Instead, it seems to provide positive reason for doubt, arising from premises which the realist accepts. It seems to reveal a tension within the realists' own total philosophical view.

Specifically, the Putnamian skeptic doesn’t just doubt that we can determinately refer to mathematical objects/physical objects/causes etc. and ask to be convinced. Rather they attempt to show that the realist can't internally coherently explain certain putative facts about reference they believe in. Putnam shows that it follows from the realist's own views on set theory, that various nonstandard interpretations 
of our 'natural number' talk exist. He suggests that the realist can't adequately explain what's wrong with these non-standard interpretations.

Accordingly, a realist answering this skeptical challenge may use their whole physical, mathematical, and metasemantic theory. They may non-question-beggingly employ terms like 'set', 'standard model' etc. (and take these to refer unproblematically) in the meta-language when giving this explanation. And they can use standard realist set theory to reason about what interpretations exist (just as Putnam does). But, of course, if no such explanation can be given, the Putnamian challenge succeeds - giving us reason to doubt our ability to determinately refer to these objects.

To help keep this dialectical situation clear, we can flesh out the conceit of the perverse interpreter (suggested above) as follow: 7 .

As before, we imagine the Putnamian skeptical challenge as a game played between the realist and a perverse interpreter who attempts to illustrate the inadequacy of the realist's theory of reference. Specifically, we will suppose that the perverse interpreter is dedicated to offering an unintended interpretation of some third party's speech, while the realist tries to force the interpreter to give the intended interpretation. Call this third party the speaker. The realist wins, i.e., succeeds in blocking the skeptical challeng $\AA^{8}$, if they can provide a principled reason for

\footnotetext{
${ }^{7}$ I take this conceit to be something like philosophical folklore.

${ }^{8}$ This is not to say that they succeed in rationally compelling the skeptic to become a realist.
} 
rejecting each of the perverse interpreter's unintended interpretations of the speaker.

In doing this, the realist is permitted to use ordinary set theory and model theory in the metalanguage to reason about what interpretations are possible (just as the skeptic does in setting up the model-theoretic challenge). They can talk about $\omega$ sequences and what standard vs. nonstandard interpretations of the speaker's number-theoretic talk exist. And they can use set theory, in an ordinary way, to reason about these interpretations. But they cannot presume that the third person whose definite reference is to be explained has any such capacity ${ }^{9}$

\footnotetext{
${ }^{9}$ This point, that we can freely refer to standard models of the natural numbers in the metalanguage when answering Putnam's challenge connects to to standard responses to (one interpretation of) Putnam's infamous and debated 'just more theory' argument in the philosophical literature.

In [16], Putnam supplements the core model-theoretic challenge sketched above with a 'just more theory' response to philosophers who attempt o use causal theories of reference to rule out unintended interpretations of our mathematical/scientific vocabulary. You might try to say that your word 'rabbit' is better interpreted as referring to rabbits than sets because you have causal contact with rabbits and not sets. But (Putnam seems to suggest) to say this is just to point out that you accept a certain further theory (apparently about the reference of your own words). And this expanded theory can still be satisfied by a model that takes all of your terms to apply only to sets. I follow Lewis [12], Devitt [5], and many others in rejecting this argument (if it is read as a development of the skeptical challenge articulated above) for the following reason. It seems to confuse the claim that a person's utterances of "rabbit" refer to rabbits because these utterances have a certain causal relationship to rabbits and not sets with the claim that they so refer because the person being interpreted accepts a bunch of sentences (which appear to articulate a causal theory of reference).

But I won't attempt to Lewis' and Devitt's arguments here. I simply want to note that even if Putnam's supplementary 'just more theory' argument fails (so causal facts can in principle be relevant to explaining reference), we are left with an interesting and troubling skeptical challenge, which has been very philosophically influential. For example, David Lewis, while rejecting the 'just more theory' argument takes Putnam's core model-theoretic skepticism seriously enough that he lists answering it as an important motivation for his theory of natural kinds 11. That core challenge is my intended topic in this paper.
} 
I will argue that (under certain physical assumptions) if the above skeptical challenge is answerable in the physical case, it is answerable in the number theoretic case as well.

Accordingly, I will imagine a scenario where the realist has already (somehow!) succeeded in blocking all unintended interpretations of the speaker's physical vocabulary. I will consider whether they can then rule out the remaining perverse interpretations which associate their number talk with some nonstandard model, while interpreting all the above mentioned physical and physical possibility language standardly.

Specifically, I will identify a certain sentence which seems to follow from claims central to our conception of the natural numbers - so that it's reasonable to expect (and I take it Putnam would grant) all acceptable interpretations of the speaker's number talk should make it come out true. Then I will argue that the Putnamian skeptic can't interpret the speaker's physical and logical vocabulary correctly (from a realist point of view), but their number talk non-standardly while making this sentence come out true. When doing this I will make a certain plausible physical assumption (IRS) and draw on this assumption when making the above argument. Note that, as IRS is only used as part of the meta-language argument about what interpretations of the speaker are possible, we may unproblematically assume mathematical realism in stating it.

However, before I do that it's first worth reviewing previous attempts to answer this challenge. 


\section{Contrast With Field and McGee}

My proposal takes inspiration from a pair of existing explanations of determinate arithmetical reference suggested by Hartry Field and Van McGee. Field and McGee each attempt to use our definite reference to certain non-mathematical vocabulary to pin down a definite interpretation (up to isomorphism) for our natural number talk.

One can think of my proposal as attempting to solve problems for Field and McGee by combining elements from each story. However, unlike Field and McGee, I'll only be arguing for the dialectically important conditional that (under certain mild assumptions) 'if we can somehow secure definite reference to certain physical/metaphysical notions, this suffices to rule out nonstandard interpretations of number talk as well'. I won't suggest that definite physical reference plays a role in the only (or best) explanation for definite mathematical reference.

\subsection{The Language Expansion Approach. In [14 McGee offers} an account of definite reference to the natural numbers which centers on what he calls 'open-endedness 10 . Open-endedness is the idea that we expect all instances of the first-order induction axiom schema to continue expressing truths in any 'logically permissible' extension of our language.

McGee argues (roughly ${ }^{11}$ ) as follows. Part of our current mathematical practice is the expectation (discussed above) that the induction

\footnotetext{
${ }^{10}$ See 15 for a related proposal.

${ }^{11} \mathrm{McGee}$ 's actual proposal is somewhat more complicated, in ways that I claim don't affect any of the criticisms discussed here. See pgs. 56-68 of [14] for the details I've elided.
} 
schema will remain true in all 'logically permissible' expansions of our language. McGee suggests that this fact helps rule out non-standard models as follows. Suppose (for contradiction) that some nonstandard model $M$ provided an acceptable interpretation of our terms 'natural number', 'successor' etc. Then there could (in some sense) be a god who can point to the non-standard model and introduce a term 'smee' which applies counter-inductively to it (i.e., 'smee' applies to a successor closed proper initial segment of the non-standard mode ${ }^{12}$. If we met such a god then we could logically permissibly extend our language by adding the term 'smee' from their language to ours. In such a case, we would still expect the induction axiom to hold for formulas involving the term 'smee' which we got from talking to this god. Therefore, interpreting us to mean a nonstandard model is unacceptable, because it any such interpretation would fail to make an instance of the induction schema (in some extended version of our language) come out true.

This proposal faces a number of worries and objections. First, it's prima facie unclear that it's metaphysically possible for a god to introduce a term like 'smee'. For instance, it's not clear how the god could definitely refer to some proper initial segment of our non-standard mode ${ }^{13}$. What can the god do to secure reference in a way we cannot? Are we to imagine a metaphysically impossible scenario where they fly

\footnotetext{
${ }^{12}$ That is, 'smee' applies to 0 , and smee $(n) \rightarrow \operatorname{smee}(S(n)$, but 'smee' doesn't apply to every (supposed) 'natural number'

${ }^{13}$ Field writes, "why can't we just say that we secure definite reference by whatever we are imagining the god to do to secure her reference?" 7 in a criticism of McGee.
} 
into the realm of abstract objects and point to each of the infinitely many elements in the initial segment one by one? ${ }^{14}$.

But McGee tries to head off all such concerns by appealing to the idea that we are committed to the first-order induction schema being true in all logically possible extensions of our language (whether these are metaphysically possible or not). He writes:

To say what individuals and classes of individuals the rules of our language permit us to name is easy: we are permitted to name anything at all. For any collection of individuals $\mathrm{K}$ there is a logically possible world - though perhaps not a theologically possible world - in which our practices in using English are just what they are in the actual world and in which $\mathrm{K}$ is the extension of the open sentence ' $\mathrm{x}$ is blessed by God'. So the rules of our language permit the language to contain an open sentence whose extension is $\mathrm{K}[14]$.

But, this insouciance comes with a cost. For appealing to something like a speaker's positive intention/determination that instances of the induction schema will continue to express truths under 'all logically possible (and logically permissible) extensions of our language' to explain how we have a determinate conception of the natural numbers is arguably question-begging. We wouldn't accept an answer to Putnam's worries that just presumed the speaker being interpreted had

\footnotetext{
${ }^{14}$ Perhaps we could imagine the god verbally providing a definite description of each object they take to be a 'natural number' in some finite stretch of time in the way discussed in the literature on supertasks [13] and then saying 'that's all'. Thanks to an anonymous reviewer for this suggestion.
} 
a determinate conception of second-order quantification. And it's not clear assuming the speaker can determinately refer to all logically possible expansions of their language, so as to explicitly form an intention to continue accepting all instances of the induction schema, is materially different. If we can somehow determinately refer to the full range of logically possible expansions of our language (where the latter are expected to witness 'all possible ways of choosing' some objects for a predicate to apply to), why can't they use the same faculty to directly intend that our second-order quantifiers range over every possible subset?:15

Instead, it might be better to interpret McGee's proposal differently (in line with his playful use of slogans like 'ignorance is strength'), as advocating a kind of negative path to determinate mathematical reference. The idea is that we commit ourselves to continued acceptance of induction under all logically possible (logically permissible) language expansions by simply being disposed to continue accepting instances of the induction schema after (logically permissible) language extensions without further doubts or checking that other conditions are satisfied. However, it seems to me that much more would need to be said to develop this picture. For example, one might dispute whether (and wonder why) having the above disposition commits one to accepting all instances of the induction schema after all logically possible logically permissible language changes rather than, e.g., merely all metaphysically possible ones.

$\overline{\left.{ }^{15} \text { See } 3\right],[8]}$ 6. 
One might also worry about whether we can rigorously cash out McGee's notion of 'logically permissible' extensions of our language 16 .

My argument will avoid both of these problems. For it makes no claims about 'all logically permissible expansions of our language', so it's not on the hook for clarifying this concept. And it won't presuppose that speakers can think about or otherwise commit themselves to accepting sentences in 'all logically possible scenarios' (or any metaphysically impossible scenarios at all).

\subsection{Appeal to the Structure of Time. In [6] Hartry Field (rather} ambivalently) proposes a different way of using physical reference to pin down reference to a unique intended natural number structure. In effect, he argues as follows. Assume that the evenly spaced points in time (starting from some specified point) form a genuine $\omega$ sequence ${ }^{17}$ (i.e., time has infinite duration and there are only a finite number of seconds between any two times). Now consider a speaker who accepts (a sentence that seems to express) this claim. Field suggests that nonstandard interpretations of this speaker's natural number talk (which interpret talk of points in time and certain temporal relations correctly) can be ruled out on the grounds that they must make this

\footnotetext{
${ }^{16}$ Sometimes, rather than talking about all logically permissible expansions of contemporary English, McGee talks about all expansions of English which are permitted by the rules of current English noting that, "the rules of English certainly permit the adjunction of additional vocabulary; indeed, we add new singular terms to the language whenever we baptize an infant or bring a new puppy home from the pound, and we add new general terms whenever we devise a new theory, discover a new species, or introduce a new product line." 14

${ }^{17}$ As usual, I'll take the claim that some plurality of objects 'forms an $\omega$ sequence' to mean that this plurality of objects (when considered under some relation $<$ ), is isomorphic to the natural number structure. So, for example it has a first element, the successor of that element and so forth.
} 
sentence come out false. However, Field's story has some limitations and also raises some concerns, which my account will avoid.

First, Field's story only pins down definite mathematical reference for people who happen to truly believe that time has a certain structure. It doesn't explain how people who keep an open mind about this matter of cosmology could refer to the natural numbers. In contrast, my story won't require that the people whose reference is to be explained have any beliefs about contingent physic: ${ }^{18}$. Thus, it will let us defend realist intuitions that even the cosmologically open-minded can definitely refer to the natural numbers.

Second, Field's story requires the assumption that time actually has a certain structure (mirroring that of the intended model of the numbers, as specified above) ${ }^{19}$. In contrast, my argument won't require that the actual laws of physics privilege the standard model of the numbers. Instead, it will merely require that a certain kind of infinite sequence of random events is physically possible. And in section 5, I will discuss some ways in which even this contingent physical assumption can be dropped.

Third, it's not clear that the mere fact that a speaker's cosmological hypothesis will come out false if we interpret their number talk non-standardly lets us rule out such interpretations as inappropriate.

\footnotetext{
${ }^{18}$ It will only require they accept that (a certain instance of) the induction schema for the natural numbers holds with physical necessity (plus some other similarly conceptually central/quasi-analytic principles about counting, which I list below). ${ }^{19}$ To bring out the weightiness of this assumption, note that physical theories on which spacetime is finite - in the sense relevant to Field's principle - have been seriously considered (c.f. [19] and 9] chapter 8). Perhaps one could even make sense of time having epochs structured like the nonstandard numbers.
} 
Certainly, most people wouldn't say that it's part of what they mean by 'the natural numbers' that the natural numbers must have a structure that mirrors the structure of time (in the sense described above). And surely, it's sometimes correct to interpret someone as holding a false belief. And this claim doesn't seem particularly conceptually central (the kind of thing any charitable interpreter must assign high priority to interpreting as expressing a truth).

More importantly, requiring all acceptable interpretations of a person's number talk to make their cosmological hypotheses (like the one above) come out true suggests the following counterintuitive consequence. If time in our world had had a nonstandard structure ${ }^{20}$ then (even if we had no evidence of this fact) our number talk would have determinately referred to a nonstandard model.

In contrast, my approach will follow McGee in appealing to a much more conceptually central aspect of our understanding of the natural numbers — the idea that it would be metaphysically and physically impossible for an instance of the induction schema (applied to physical objects) to fail — which would be violated if our natural number talk didn't refer to the standard model. This lets us avoid the unappealing idea that, if the physical laws had been undetectably different (while all our experiences and utterance dispositions remained the same) our number talk we would have determinately referred to some nonstandard model.

\footnotetext{
${ }^{20}$ In talking about scenarios where 'time has a nonstandard structure', I mean to consider scenarios in which the evenly spaced moments (e.g., the ticks of a perpetual clock) after a certain point form a nonstandard model of first-order Peano Arithmetic.
} 


\section{My Proposal}

With all this background in place, I will now argue for the core thesis of this paper: if a certain plausible physical assumption holds and we have intended realist reference to certain physical notions, we can resist Putnam's argument against determinate reference to the intended natural number structure.

Remember that, as I outlined above, our goal is to identify a sentence $\square_{P}$ COIN INDUCT that (unlike the cosmological hypothesis used in Field's account) is treated as sufficiently central to our conception of 'the natural numbers' that interpretations of our number talk can plausibly be ruled out for failing to make this sentence come out true. I'll argue that (given a plausible physical assumption) no interpretation makes $\square_{P}$ COIN INDUCT come out true while interpreting all physical and logical vocabulary as the realist intends, and our number talk as referring to some nonstandard model of PA.

More specifically, the structure of this argument will be as follows:

- Identify a claim COIN INDUCT whose physical necessity is treated as sufficiently conceptually central that failing to make $\square_{P}$ COIN INDUCT (where $\square_{P}$ is a physical necessity operator) come out true can rule out candidate interpretations of our 'number' talk ${ }^{21}$ Specifically, COIN INDUCT will conjoin the following (first-order English) claims.

\footnotetext{
${ }^{21}$ This is not to say that all acceptable interpretations must make all sentences treated as central to the speaker's conception of some concept come out true. If the collection of seemingly conceptually central truths about the numbers were incoherent so that no interpretation could make them all come out true, the situation would be different .
} 
HEADS INDUCT: An instance of the first-order induction schema as applied to the property $Q(n)$ asserting that there is an $n$-th coin flip and it landed heads.

COUNTING RULES: A collection of conceptually central principles like 'For all objects $x$ and numbers $n$, if $x$ is the $n$-th coin flip, then $x$ is a coin flip.'

$\mathbf{P A}^{-}$: The usual Peano Axioms of arithmetic without the induction schema.

- Identify (by stating in the meta-language) and make a certain plausible assumption about what's physically possible (IRS).

- Note that if IRS holds then no non-standard interpretation of the speaker's number talk can make $\square_{p}$ COIN INDUCT come out true, while interpreting their expression ' $\square_{p}$ ' and certain other physical vocabulary as the realist intends.

- Conclude that (if this plausible physical assumption holds) the speaker's securing definite realist reference for all their physical vocabulary would suffice to block all Putnamian nonstandard interpretations of their 'number' talk.

In a sense, my proposal will use the fact that it's physically possible for any subset of an infinite independent sequence of coin flips to land heads to take the place of second-order quantification in ruling out nonstandard models of number theory.

4.1. Assumptions. To more rigorously develop the argument above, let me first give a definition of the kind of interpretations at issue in this argument. This definition simply cashes out the idea that we're 
taking definite reference to physical necessity for granted and imposes some basic constraints on our would-be perverse interpreter.

I will say that an interpreter is well behaved just if they satisfy the following criteria:

- They select a single model as the referent of our concept 'natural number' in all physically possible worlds.

- They don't tamper with the extension of physical vocabulary like 'coin flip', 'landed heads' or 'temporally before' at any of these possible worlds.

- They interpret all first-order logical vocabulary and the physical necessity operator standardly. So, for example, they will take the speaker's existential quantifier and the physical necessity operator $\square_{p}$ to contribute to truth conditions in the usual fashion.

- They interpret all sentences we treat as conceptually central to our understanding of the natural numbers ${ }^{22}$ (on par with the Peano Axioms) as expressing truths.

4.2. Conceptually Central Claims. To introduce the key sentences at issue, consider how we use numbers to talk about temporally (or otherwise) ordered sequences of events. We talk about, 'the 4th U.S. President', 'the 37th successful rickrolling', or 'the 3rd coin flip (in the history of the universe)'.

\footnotetext{
${ }^{22}$ Note that being conceptually central in the sense at issue here does not require being indubitable and could be a matter of degree 20 .
} 
Given a type of physical event, 'coin flip' 23 , we (and hence the speaker) have, or can easily define ${ }^{24}$, a relation 'countflip(n,x)' between coin flip events and events, which holds when ' $x$ is the $n$-th coin flip' (counting from 0$)$. We take this relation to relate 0 to the temporally first coin flip event in the history of the universe (if there is one) 1 to the temporally next coin flip event and so on. Speaking in these terms, we (and hence the speaker) take the following claims to hold with physical (and indeed metaphysical) necessity.

- An object $x$ is the 0th coin flip, iff $x$ is a coin flip and all other coin flips happen after $x^{26}$

- If $x$ is the $n$th coin flip, then $y$ is the $n+1$-th coin flip iff $y$ occurs after $x$ and no other coin flip occurs between $x$ and $y 27$. - Only coin flips can be the $n$th coin flip 28 .

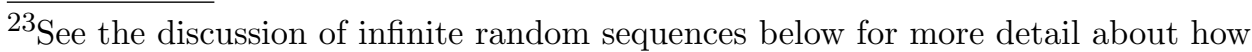
I want to understand this notion.

${ }^{24}$ Admittedly for the purpose of general logical analysis of language, we'll probably want to formalize ordinary counting claims in a more abstract way. For we'll want to account for our ability to say 'there's a 17-th $x$ such that $\phi(x)$ (considered under ordering $\rho$ )' for infinitely many different predicates $\phi$ (and ordering $\rho$ ) while speaking a finitely learnable language.

${ }^{25}$ In some cases which coin flip counts as the temporally first one may be relative to a choice of a reference frame, but this complication poses no difficulty. We may simply work relative to some foliation or, alternatively, restrict our attention to those possible worlds in which there is a unique first coin flip.

${ }^{26}$ That is, $(\forall x)[\operatorname{count} f l i p(0, x) \leftrightarrow$ coinflip $(x) \wedge(\forall y)(\operatorname{count} f l i p(y) \rightarrow$ before $(x, y) \vee$ $x=y)$ )]. Here I count up from 0 rather than 1 , for the sake of simplicity. Also, by writing this formula with 0 , I abbreviate the corresponding claim about the unique object satisfying some definite description of 0 in terms of $\mathbb{N}, S$, e.g., 'the unique object that has a successor but isn't a successor'.

${ }^{27}$ That is,

$$
\begin{aligned}
(\forall n)(\forall x)(\forall y)(\mathbb{N}(n) & \wedge \text { countflip }(n, x) \rightarrow[(\operatorname{countflip}(S(n), y) \leftrightarrow \\
\operatorname{coinflip}(y) & \wedge \text { before }(x, y) \wedge(\forall z) \neg(\operatorname{coinflip}(z) \wedge \text { before }(x, z) \wedge \text { before }(z, y))]
\end{aligned}
$$$$
{ }^{28} \text { That is }(\forall x)(\exists n)(\text { countflip }(n, x) \rightarrow \text { coinflip }(x)) \text {. }
$$ 
- No two distinct numbers correspond to the same coin flip ${ }^{29}$

Call the conjunction of all the principles above COUNTING RULES. Then, I take it, $\square_{P}$ COUNTING RULES is (or follows from) principles treated as 'conceptually core' truths, which (just as much as the Peano Axioms) all acceptable interpretations of our 'number', 'coinflip' and 'countflip' talk must satisfy 30 .

Second, we take induction to hold for all predicates in our language, including $Q(n)$ "there is an $n$-th coin flip, and the $n$-th coin flip comes up heads," $Q(n) \stackrel{\text { def }}{=}(\exists x)($ countflip $(n, x) \wedge$ heads $(x))$. And we can state an instance of the induction schema for $Q(n)$ as follows.

\section{(HEADS INDUCT)}

$$
Q(0) \wedge(\forall n)[Q(n) \rightarrow Q(S(n))] \rightarrow(\forall n)[\mathbb{N}(n) \rightarrow Q(n)]
$$

Indeed, we think it's (logically, physically, and metaphysically) impossible for any property to apply to 0 and the successor of every number it applies to without applying to all numbers. So we and the speaker will both take HEADS INDUCT to hold with physical necessity.

So let me define COIN INDUCT to be the conjunction of the induction schema for $Q(n)$ (the $n$-th coin flip landed heads) and the rules for counting coin flips. That is,

\footnotetext{
${ }^{29}$ That is $(\forall n)(\forall m)(\operatorname{coinflip}(n, x) \wedge \operatorname{coinflip}(m, x) \rightarrow m=n)$

${ }^{30}$ Of course, to say that COUNTING RULES is a conceptually central truth for us isn't to say that in some other context or language we couldn't instead start counting coin flips from 1 rather than 0. Also, I've only suggested the above as conceptually central principles for counting all coin flips. One could obviously count all coin flips after Tuesday as well and we'd have to modify COUNTING RULES if we wished to describe counting those events.
} 


\section{$($ COIN INDUCT $)$ HEADS INDUCT $\wedge$ COUNTING RULES $\wedge P A^{-}$}

I take it that $\square_{P}$ (COIN INDUCT) is (or follows from) principles which we (and hence the speaker) treat as conceptually core. The Putnamian skeptic allows that any acceptably charitable interpretation must make the axioms of Peano Arithmetic true. And it seems equally clear that any acceptable interpretation must make $\square_{P}(\mathrm{COIN}$ INDUCT) come out true. For this principle simply says that (an instance of) natural number induction and certain seemingly analytic truths about counting coin flips hold with physical necessity.

This completes my identification of the specific object language sentence ( $\square_{P}$ COIN INDUCT) whose truth our well-behaved interpreter can't guarantee while interpreting our number talk non-standardly.

4.3. Infinite Random Sequences. Now let's turn to the task of showing (by set theoretic reasoning in the meta-language) that our perverse interpreter can't make $\square_{P}$ COIN INDUCT true while obeying the rules above without interpreting number talk standardly. My argument begins with the following plausible, though not indubitable, physical assumption.

Infinite Random Sequence Assumption (IRS): It is physically possible to have a series of independent objectively random events with two possible outcomes and the following combination of features. The events are linearly 
ordered in time ${ }^{31}$ there's a first event but no final event and every event in the series has a temporal successor, i.e., for any event $x$ there is some other event $y$ occurring after $x$ such that no event $z$ occurs between $x$ and

\section{$y$.}

Informally, one can think of the events whose possibility IRS asserts as being like the ticks of an indestructible watch which never needs repair or winding. There is a first tick, each tick is followed by a unique next tick, and there is no tick after which the watch breaks down.

To motivate this principle, note that it is only asserting that it is physically possible to repeatedly perform (independent) textbook spin measurements on an electron ${ }^{32}[1]$ or some other equivalent process. Also, plausibly, the laws of physics don't rule out time continuing infinitely into the future (though possibly having non-standard 'length'). I will abstract away from all details about these objectively random events in what follows, and simply refer to each event as 'a coin flip' and the two outcomes as 'heads' and 'tails 33 .

\footnotetext{
${ }^{31}$ That is, for any distinct events $x, y$ in the series, either $x$ occurs before $y$ or $y$ occurs before $x$. Moreover, from the point of view of relativistic physics, the measurements are separated by time-like intervals $(x$ is in the future light cone of $y$ or vice versa) so all observers agree on their order. Given these constraints, it is safe to simply work relative to some foliation and ignore relativistic complications for the remainder of the paper. Thanks to Peter Gerdes for help developing this point and other details about physics.

${ }^{32}$ That is, perform a spin measurement along the $x$-axis on an electron whose spin has just been measured (and thus collapsed) along the $y$-axis.

${ }^{33}$ Even if you are not convinced that infinite random sequences of the kind discussed above are physically possible, they are surely plausible enough that it's uncomfortable for any philosophical view to be committed to ruling them out a priori. Also note that ultimately I will only use IRS to establish that it's physically possible for the events satisfying some physical predicate (having a determinate extension) in
} 
Finally, note that we might need to use mathematical vocabulary to state the probability and independence claims in IRS. As discussed in $\$ 2.2$, I don't take this to be a problem for a realist aiming to defend herself from Putnamian skepticism. For I will only be appealing to the assumption IRS (and using the mathematical vocabulary needed to state it) in the metalanguage, to argue that that certain perverse interpretations of the speaker are impossible.

To put the point another way, while we (working in the metalanguage) might use mathematical language, my argument only assumes that the speaker can successfully refer to the full range of physical possibilities with their 'It is physically possible that...' operator ${ }^{34}$

4.4. Ruling out Nonstandard Models. With these to bits of background in place, we can finally foil the (well behaved) perverse interpreter.

By our assumption IRS, there's a physically possible world $w_{\omega}$ where infinitely many independent random coin flips (linearly ordered by the relation ' is temporally before') take place. This sequence of coin flips need not itself form an $\omega$ sequence (from the point of view of our background set theory), but it has an initial segment which does ${ }^{35}$. By

our current language to form an $\omega$ sequence (with the relation of 'temporally before' playing the role of $<$ ). So readers who already accept this claim could replace IRS with a direct assertion of the physically possibility of such a physically definable $\omega$ sequence. However, I feel IRS provides compelling reason to accept this conclusion. ${ }^{34}$ Note that it might take quite different mathematical resources to refer to the notion of physical possibility (as needed to ask questions about whether it would be physically possible for a pig to fly, or for there to be a perpetual motion machine) vs. to state a scientific theory which picks out the set of physically possible worlds in some other terms.

${ }^{35}$ Using ordinary background set theory to reason about what models and interpretations exist just as Putnam does, we can prove that any infinite discrete linear 
the independence and randomness of the coin flips, it follows that there are physically possible scenarios corresponding to all combinatorially possible outcomes for each coin flip.

So there's some physically possible world $w_{\omega}$, where exactly an initial $\omega$ sequence of these coin flips come up heads.

However, we can now show that our constraints on the perverse interpreter ensure that she takes $Q(n) \stackrel{\text { def }}{=}(\exists x)(\operatorname{countflip}(n, x) \wedge$ heads $(x))$ to hold for just those $n$ in the standard initial segment of the nonstandard referent of the natural numbers - so she's forced to say HEADS INDUCT comes out false at this world.

Importantly, we can show this by an argument doesn't questionbeggingly assume that the speaker can somehow pick out and refer to worlds at which only an initial $\omega$ sequence of coin flips come up heads. It only requires that the speaker can secure definite realist reference for the physical vocabulary 'coin flip', 'heads ' etc. mentioned above (at the actual world and a suitable range of physically possible worlds) and $\square_{P}$, while $\square_{P}$ COIN INDUCT as a conceptually central truth.

We can show, as follows, that no interpretation (recognized by our background set theory) can make HEADS INDUCT, COUNTING RULES and $\mathrm{PA}^{-}$come out true at $w_{\omega}$, while taking 'coinflip()', 'heads()' and before() to have their intended extension, but number talk to apply to some nonstandard model at $w_{\omega}$.

Consider how one can interpret 'countflip()' as applying to this world $w_{\omega}$, where exactly the initial $\omega$ sequence of coin flips comes up every model of $P A^{-}$also includes an initial $\omega$ sequence. 
heads. To satisfy the principles governing 'countflip()' in COUNTING RULES, 0 has to be assigned to the temporally first coin flip in $w_{\omega}, 1$ to the next, and so on, for all the objects in the standard initial segment of the nonstandard model (note that every nonstandard model of $P A^{-}$has a standard initial segment). This 'uses up' all the coin flips landing heads. Since, by COUNTING RULES 'countflip()' pairs 'numbers' with coin flips 1-1, no coin flip can be reused.

Accordingly, a perverse interpreter must take 'countflip()' pair all and only the 'true' (i.e., standard) natural numbers with coin flips landing heads. Thus, their interpretation must make ' $Q(0)$ ' and 'whenever $Q$ applies to some number $n$ it also applies to $Q(n+1)^{\prime}$ true. But as their interpretation takes 'the numbers' to refer to a larger structure than this standard initial segment, it follows that their interpretation also makes ' $\forall n Q(n)$ ' false. Thus, their interpretation renders HEADS INDUCT false at this physically possible world, and hence $\square_{p}$ COIN INDUCT false (full stop).

So, to summarize, if IRS is true then (provided 'coinflip()', 'heads()' and before() have their usual interpretation), either 'natural number' is interpreted standardly or some part of COIN INDUCT doesn't hold with physical necessity.

\section{Generalizing the Point By Dropping/CHAnging SOME}

\section{ASSUMPTIONS}

So much for my core argument. Above I have argued that (given the plausible physical assumption IRS mentioned above) one cannot interpret physical vocabulary standardly and number talk nonstandardly 
without making some applied mathematical sentences we treat as obvious conceptual truths about the natural numbers come out false. Let me end by pointing out some quick correlaries and generalizations.

First, the argument above works just the same if we replace appeal to physical possibility $\square_{p}$ with an appeal to metaphysical possibility $\square_{m}$. And it is surely metaphysically possible for there to be an infinite random sequence of independent coin flips (as per IRS). So, if you assume that we (somehow) have a determinate realist grasp on metaphysical possibility and the basic physical vocabulary above, this also suffices to block the Putnamian skeptic's argument ${ }^{36}$.

Second, we don't need to assume possession of a completely determinate notion of physical/metaphysical possibility for the argument above. It's fine if our conceptions of the limits of physical/metaphysical possibility are underspecified in various ways - provided that all acceptable precisifications of these notions agree in classifying some world like $w_{\omega}$ as physically possible. And this is something which many existing reasons for thinking that our notions of physical/metaphysical possibility aren’t fully definite don't call into question.

Third, we might be able to eliminate premises about determinate reference to physical/metaphysical possibility from my argument all together, by instead appealing to the idea that principles of charity [4] favor making speakers out to be rational/justified as well as attributing

\footnotetext{
${ }^{36}$ We could also make a similar argument without appealing to the notion of random events at all. We could just appeal directly the intuition that (from a realist point of view) it would be metaphysically possible for there to be an $\omega$ sequence of coin flips which came up heads. This seems strongly motivated by the intuition that infinite collections of some kind are physically possible plus general Humean Recombination intuitions.
} 
them more true beliefs. For, (arguably) if we don't interpret people to be talking about the standard model of the natural numbers, then their (approximately) a priori confidence in instances of the induction schema will look like unjustified dogmatism. The existence of physical $\omega$ sequences as in world $w_{\omega}$, seems like something we shouldn't rule out a priori. But (by the argument above) any nonstandard interpretation of our number talk would make induction fail in this epistemically possible scenario.

So accepting principles like COIN INDUCT as necessary truths knowable a priori ${ }^{37}$, looks rational on a standard interpretation of the numbers, but irrational on any nonstandard interpretation of our number talk 38

\section{Conclusion}

In this paper, I have argued that if we have determinate realist reference for certain pieces of physical vocabulary, Putnamian model theoretic arguments that we can't determinately refer to the natural numbers are blocked. Specifically, I have argued that (given certain plausible assumptions), merely securing determinate reference to physical possibility and certain other physical concepts suffices to rule out all non-standard models of our number-theoretic talk as well.

Let me close with three notes of humility. First (as noted above), I don't claim to have answered Putnam's challenge or explained how

\footnotetext{
${ }^{37}$ Here I mean to refer to our a priori confidence in $\square_{p}$ COIN INDUCT.

${ }^{38}$ See 2 for development of an idea along these lines in a different philosophical context (attacking certain anti-realist views, rather than answering Putnamian skepticism).
} 
we can determinately grasp mathematical concepts. I have only argued for a (dialectically important) conditional claim. If one could somehow answer Putnam's challenge regarding physical concepts, this would provide a principled reason for rejecting nonstandard interpretations of our number talk as well. I've provided no argument that we should respond to this fact by rejecting Putnam's arguments against physical and number-theoretic determinate reference rather than, e.g, taking them to succeed in both cases.

Second, remember that Putnam's model theoretic argument seemed to identify an internal problem for the realist. He notes that, even from the realist perspective (i.e., granting use of familiar realist set theory in the metalanguage) certain perverse interpretations exist, and challenges the realist to explain what makes all the unintended interpretations wrong. I've argued that, if certain assumptions hold, this challenge can be answered; we can internally coherently explain what's wrong with all the unintended interpretations our background set theory acknowledges. However, this doesn't prevent fans of determinate physical reference from rejecting determinate reference to the natural numbers for some other reason than Putnam's model theoretic argument.

Third, although I have argued that (if we can somehow definitely refer to it) leveraging physical possibility and accepting principles like $\square_{p}(\mathrm{COIN}$ INDUCT) offers a route to grasping a definite concept of the natural numbers, I don't mean to suggest that this is the only or primary way that we can grasp such a concept. For another thing, it 
would be strange if our possession of a definite conception of the natural

number structure depended on our beliefs about physical (or metaphysical) possibility. Thus, I suspect that another - rather different- style of answer to Putnam's challenge must also be possible.

\section{REFERENCES}

[1] David Z. Albert. Quantum Mechanics and Experience. Harvard University Press, 2009.

[2] Sharon Berry. $\Sigma_{1}^{0}$ Soundness isn't Enough. British Journal of Philosophy of Science, Forthcoming.

[3] Tim Button and Sean Walsh. Structure and Categoricity: Determinacy of Reference and Truth Value in the Philosophy of Mathematics. Philosophia Mathematica, 24(3):283-307, 2016.

[4] Donald Davidson. Three Varieties of Knowledge. In A. Phillips Griffiths, editor, Royal Institute of Philosophy Supplement, pages 153-166. New York: Cambridge University Press, 1991.

[5] M. Devitt. PUTnAM, H., "Meaning and the Moral Sciences". Australasian Journal of Philosophy, 58(n/a):390, 1980.

[6] Hartry Field. Which undecidable mathematical sentences have determinate truth values? Truth in Mathematics, pages 291-310, 1998.

[7] Hartry Field. Truth and the Absence of Fact. Oxford University Press, 2001.

[8] K. Gödel. Über formal unentscheidbare sätze der principia mathematica und verwandter systeme i. Monatshefte für Mathematik, 38(1):173-198, 1931.

[9] S. Hawking. A Brief History of Time. Random House Publishing Group, 2011.

[10] Harold T. Hodes. Logicism and the Ontological Commitments of Arithmetic. Journal of Philosophy, 81(3):123-149, 1984.

[11] David Lewis. New Work for a Theory of Universals. Australasian Journal of Philosophy, 61(4):343-377, 1983. 
[12] David Lewis. Putnam's Paradox. Australasian Journal of Philosophy, 62(3):221-236, 1984.

[13] David Malament. Science Without Numbers by Hartry H. Field. Journal of Philosophy, 79(9):523-534, 1982.

[14] Vann McGee. How We Learn Mathematical Language. Philosophical Review, 106(1):35-68, 1997.

[15] Charles Parsons. The Uniqueness of the Natural Numbers. The Jerusalem Philosophical Quarterly, 39:13-44, 1990.

[16] Hilary Putnam. Realism and Reason. Proceedings and Addresses of the American Philosophical Association, 50(6):483-498, 1977.

[17] Hilary Putnam. Models and Reality. Journal of Symbolic Logic, 45(3):464-482, 1980.

[18] Jared Warren and Daniel Waxman. A metasemantic challenge for mathematical determinacy. Synthese, 197(2):477-495, 2020.

[19] Wikipedia contributors. Big crunch — Wikipedia, the free encyclopedia, 2019. [Online; accessed 3-March-2019].

[20] Timothy Williamson. The Philosophy of Philosophy (The Blackwell / Brown Lectures in Philosophy). Wiley-Blackwell, Oxford, 2008. 